05,11

\title{
Критическая релаксация трехмерной полностью фрустрированной модели Изинга
}

\author{
(C) В.А. Мутайламов ${ }^{1}$, А.К. Муртазаев ${ }^{1,2}$ \\ ${ }^{1}$ Институт фризики ДагНЦ РАН, \\ Махачкала, Россия \\ 2 Дагестанский государственный университет, \\ Махачкала, Россия \\ E-mail: vadim.mut@mail.ru
}

\begin{abstract}
Методом коротковременной динамики изучена критическая релаксация из низкотемпературного упорядоченного состояния трехмерной полностью фрустрированной модели Изинга на простой кубической решетке. Исследованы системы кубической формы с периодическими граничными условиями и линейными размерами $L=32,64,96$ и 128 в каждом кристаллографическом направлении. Вычисления проводились методом Монте-Карло с использованием стандартного алгоритма Метрополиса. Рассчитаны значения статических критических индексов намагниченности и радиуса корреляции, а также величины динамического критического индекса.
\end{abstract}

DOI: 10.21883/FTT.2018.06.45984.12M

\section{1. Введение}

Исследование динамических критических свойств спиновых систем является одной из актуальных задач современной статистической физики и физики фазовых переходов. К настоящему времени в этой области достигнуты существенные успехи, обусловленные главным образом теоретическими и экспериментальными исследованиями. Тем не менее построение строгой и последовательной теории динамических критических явлений на основе микроскопических гамильтонианов является одной из центральных проблем современной теории фазовых переходов и критических явлений, которая все еще далека от своего решения.

В последнее время для изучения критической динамики моделей магнитных материалов стал успешно применяться метод коротковременной динамики (shorttime dynamic) [1-5], в котором в рамках модели $A$ (классификация классов универсальности динамического критического поведения Хальперина и Хоэнберга [6]) исследуется критическая релаксация магнитной модели из неравновесного состояния в равновесное. Традиционно считается, что универсальное скейлинговое поведение существует только в состоянии термодинамического равновесия. Однако было показано, что универсальное скейлинговое поведение для некоторых динамических систем может реализовываться на ранних этапах их временно́й эволюции из высокотемпературного неупорядоченного состояния в состояние, соответствующее температуре фазового перехода [7]. Такое поведение реализуется после некоторого отрезка времени, который является достаточно большим в микроскопическом смысле, но остается малым в макроскопическом. Аналогичная картина наблюдается и в случае эволюции системы из низкотемпературного упорядоченного состояния $[1,2]$.

Ранее нами методом коротковременной динамики исследована критическая релаксация из низкотемператур- ного упорядоченного состояния трехмерной полностью фрустрированной модели Изинга на простой кубической решетке для линейного размера $L=64$ [8]. В настоящей работе для оценки влияния размеров систем на получаемый результат мы исследовали критическую релаксацию данной модели для линейных размеров $L=32,64,96$ и 128. Кроме того, мы улучшили статистику вычислений и изменили метод определения критической температуры. Это позволило нам увеличить точность определения значений критической температуры и критических индексов.

\section{2. Методика исследований}

Используя метод ренормгруппы, авторы [7] показали, что вдали от точки равновесия после микроскопически малого отрезка времени для $k$-го момента намагниченности реализуется скейлинговая форма

$$
M^{(k)}\left(t, \tau, L, m_{0}\right)=b^{-k \beta / \nu} M^{(k)}\left(b^{-z} t, b^{1 / \tau} \tau, b^{-1} L, b^{x_{0}} m_{0}\right),
$$

где $M^{(k)}-k$-й момент намагниченности; $t-$ время; $\tau$ - приведенная температура; $L-$ линейный размер системы; $b$ - масштабный фактор; $\beta$ и $v-$ статические критические индексы намагниченности и радиуса корреляции; $z$ - динамический критический индекс; $x_{0}-$ новый независимый критический индекс, определяющий скейлинговую размерность начальной намагниченности $m_{0}$.

При старте из низкотемпературного упорядоченного состояния $\left(m_{0}=1\right)$ в критической точке $(\tau=0)$, полагая $b=t^{1 / z}$ в уравнении (1), для систем с достаточно большими линейными размерами $L$ теория предсказывает степенное поведение намагниченности в коротковременном режиме

$$
M(t) \sim t^{-c_{1}}, \quad c_{1}=\frac{\beta}{v z} .
$$


Логарифмируя обе части уравнения (2) и беря производные по $\tau$ при $\tau=0$, получаем степенной закон для логарифмической производной

$$
\left.\partial_{\tau} \ln M(t, \tau)\right|_{\tau=0} \sim t^{-c_{1}}, \quad c_{l_{1}}=\frac{1}{v z} .
$$

Для куммулянта Биндера $U_{L}(t)$, рассчитываемого по первому и второму моментам намагниченности, теория конечно-размерного скейлинга дает следующую зависимость при $\tau=0$ :

$$
U_{L}(t)=\frac{M^{(2)}}{(M)^{2}}-1 \sim t^{c_{U}}, \quad c_{U}=\frac{d}{z},
$$

где $d$ - размерность системы.

Таким образом, в ходе одного численного эксперимента метод коротковременной динамики позволяет с использованием соотношений (2)-(4) определить значения трех критических индексов $\beta, v$ и $z$. Кроме того, зависимости (2), построенные при различных значениях температуры, позволяют определить величину $T_{c}$ по их отклонению от прямой линии в двойном логарифмическом масштабе.

\section{3. Модель}

С использованием данного метода нами исследована критическая релаксация из низкотемпературного упорядоченного состояния трехмерной полностью фрустрированной модели Изинга на простой кубической решетке. Впервые данная модель была предложена Вильяном [9] в двумерном случае на квадратной решетке для описания спиновых стекол. В дальнейшем она была обобщена Бланкштейном [10] для трехмерного случая. Схематически модель показана на рис. 1.

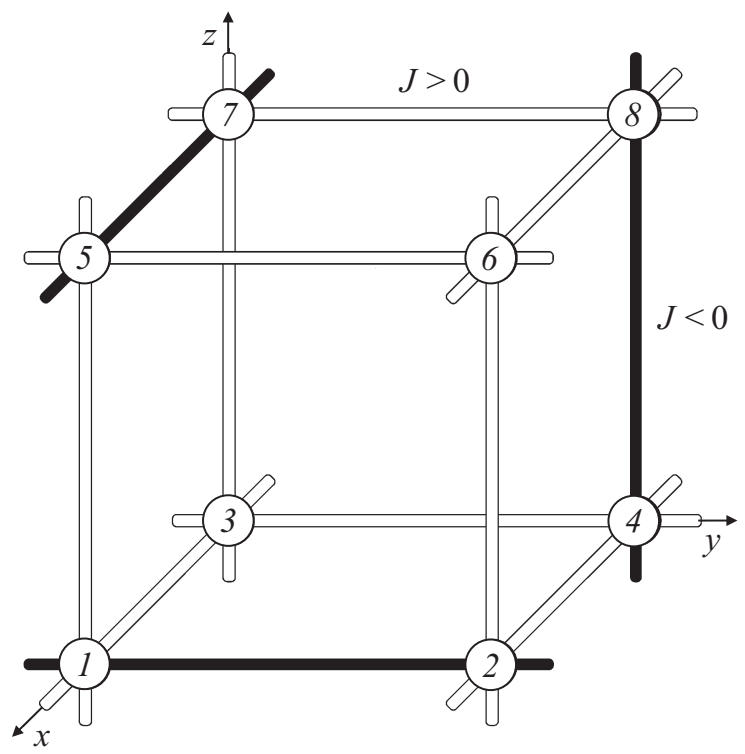

Рис. 1. Полностью фрустрированная модель Изинга на простой кубической решетке. Белым цветом отмечены ферромагнитные связи $(J>0)$, черным - антиферромагнитные $(J<0)$.
Интерес к данной модели обусловлен тем, что при изучении фрустрированных систем основное внимание уделяется моделям на треугольной и гексагональной решетках, тогда как свойства моделей на кубической решетке исследованы мало. В том числе, практически не изучено динамическое критическое поведение данных систем.

Гамильтониан фрустрированной модели Изинга может быть представлен в виде

$$
H=-\frac{1}{2} \sum_{\langle i, k\rangle} J_{i k} S_{i} S_{k}, \quad S_{i}= \pm 1,
$$

где $S_{i}-$ изинговский спин в узле решетки $i ; J_{i k}$ обменное взаимодействие между спинами для ферромагнитных $(J>0)$ и антиферромагнитных $(J<0)$ связей. Фрустрации в этой модели обусловлены конкуренцией обменных взаимодействий [9].

\section{4. Результаты}

Нами исследованы системы кубической формы с периодическими граничными условиями, содержащие $L \times L \times L$ элементарных ячеек. Вычисления проводились для систем с линейными размерами $L=32,64,96$ и 128 , содержащими соответственно $N=32768,262144$, 884736 и 2097152 спинов, методом Монте-Карло с использованием стандартного алгоритма Метрополиса. Релаксация системы осуществлялась из начального низкотемпературного полностью упорядоченного состояния со стартовым значением намагниченности $m_{0}=1$ в течение времени $t_{\max }=1000$, где в качестве единицы „времени“ брался один шаг расчета методом Монте-Карло на спин. Релаксационные зависимости для каждого значения температуры вычислялись $n$ раз, полученные данные усреднялись. Величина $n$ выбиралась в зависимости от размера системы и составляла $n=10^{5}$ для $L=32$ и $L=64, n=8 \cdot 10^{4}$ для $L=96$ и $n=7 \cdot 10^{4}$ для $L=128$. Отметим, что в [8] мы использовали значение $n=5 \cdot 10^{4}$ для линейного размера $L=64$.

Для каждого линейного размера вычисления проводились при пяти значениях температуры в окрестностях точки фазового перехода. Значения этих температур в единицах обменного интеграла $k_{b} T /|J|$ приведены в табл. 1. Величина $T_{3}$ выбиралась максимально близкой к величине $T_{c}$. По полученным результатам методом

Таблица 1. Значения температур, при которых проводились вычисления

\begin{tabular}{r|c|c|c|c|c}
\hline$L$ & $T_{1}$ & $T_{2}$ & $T_{3}$ & $T_{4}$ & $T_{5}$ \\
\hline 32 & 1.3387 & 1.3437 & 1.3487 & 1.3537 & 1.3587 \\
64 & 1.3387 & 1.3437 & 1.3487 & 1.3537 & 1.3587 \\
96 & 1.3389 & 1.3439 & 1.3489 & 1.3539 & 1.3589 \\
128 & 1.3387 & 1.3437 & 1.3487 & 1.3537 & 1.3587
\end{tabular}


Таблица 2. Значения критических индексов и критических температур

\begin{tabular}{r|c|c|c|c|c|c|c}
\hline \multicolumn{1}{c|}{$T_{c}$} & $c_{1}$ & $c_{l 1}$ & $c_{U}$ & $\beta$ & $v$ & $z$ \\
\hline 32 & $1.34362(2)$ & $0.142(3)$ & $0.702(3)$ & $1.186(7)$ & $0.202(4)$ & $0.563(4)$ & $2.530(7)$ \\
64 & $1.34872(3)$ & $0.184(3)$ & $0.839(3)$ & $1.343(7)$ & $0.220(4)$ & $0.534(4)$ & $2.234(7)$ \\
96 & $1.34870(3)$ & $0.183(3)$ & $0.839(3)$ & $1.345(7)$ & $0.218(4)$ & $0.534(4)$ & $2.230(7)$ \\
128 & $1.34873(3)$ & $0.183(3)$ & $0.839(3)$ & $1.344(7)$ & $0.218(4)$ & $0.534(4)$ & $2.232(7)$
\end{tabular}

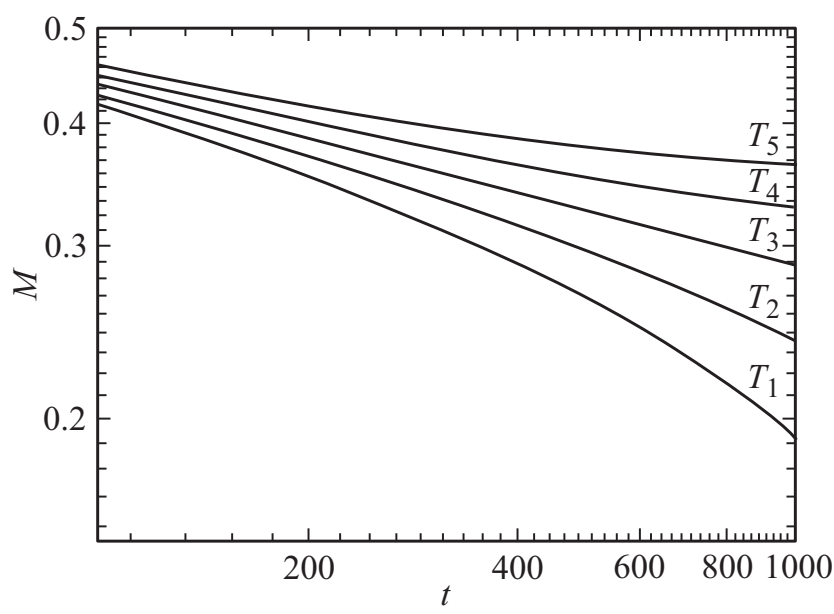

Рис. 2. Зависимость намагниченности от времени при пяти значениях температур для линейного размера $L=64$. Величины температур указаны в табл. 1 .

наименьших квадратов проводилась аппроксимация зависимостей (2), (3) и (4) в интервале температур от $T_{1}$ до $T_{5}$ с шагом $\Delta T=10^{-5}$.

Критическая температура определялась по зависимости намагниченности от времени (2), которая в точке фазового перехода должна представлять собой прямую линию в двойном логарифмическом масштабе. Отклонение от прямой линии определялось методом наименьших квадратов. За критическую принималась температура, при которой это отклонение было минимальным. Рис. 2 демонстрирует типичную зависимость намагниченности от времени при различных значениях температуры (здесь и далее все величины приведены в условных единицах). Найденные таким образом значения критических температур для всех линейных размеров приведены в табл. 2.

В работе [8] мы анализировали температурные кривые намагниченности с шагом $\Delta T=10^{-4}$ в области точки фазового перехода, после чего проводили прямые вычисления при найденной критической температуре. Изменение методики определения критической температуры в настоящей работе позволило увеличить точность наших вычислений.

Полученные нами зависимости намагниченности, ее логарифмической производной и куммулянта Биндера от времени в критической точке в двойном логарифмическом масштабе в интервале времени $t=[10 ; 1000]$ представлены соответственно на рис. 3,4 и 5. Сплошными линиями на графиках представлены результаты для линейных размеров $L=64,96$ и 128. Пунктирными линиями представлены результаты для линейного размеpa $L=32$.

Как видно из рис. 3 и 4, графики для $L=64,96$ и 128 практически совпадают друг с другом. Так, например, разница в значениях $M$ в каждый момент времени $t$ для всех этих размеров $L$ составляет порядка $10^{-3}$. При этом

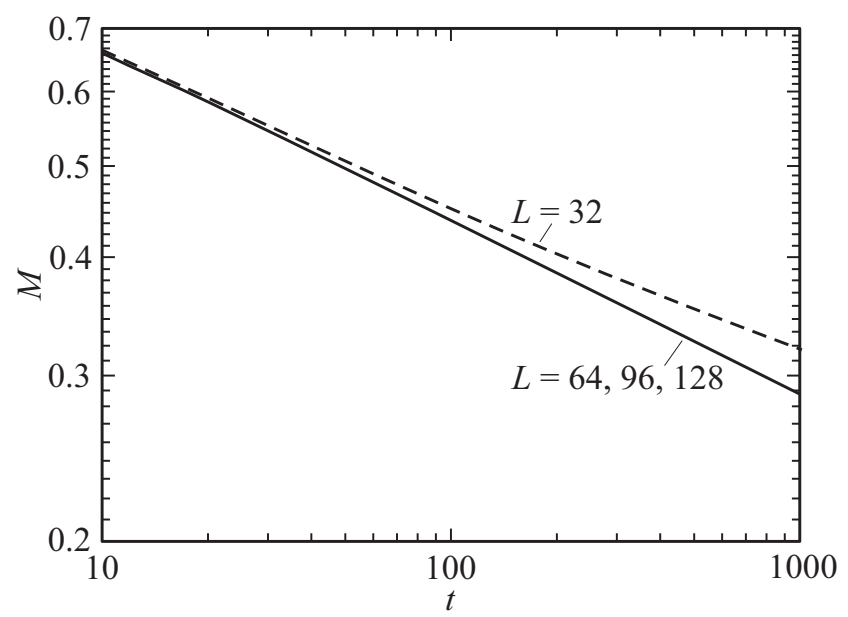

Рис. 3. Зависимость намагниченности от времени в точке фазового перехода для всех линейных размеров $L$.

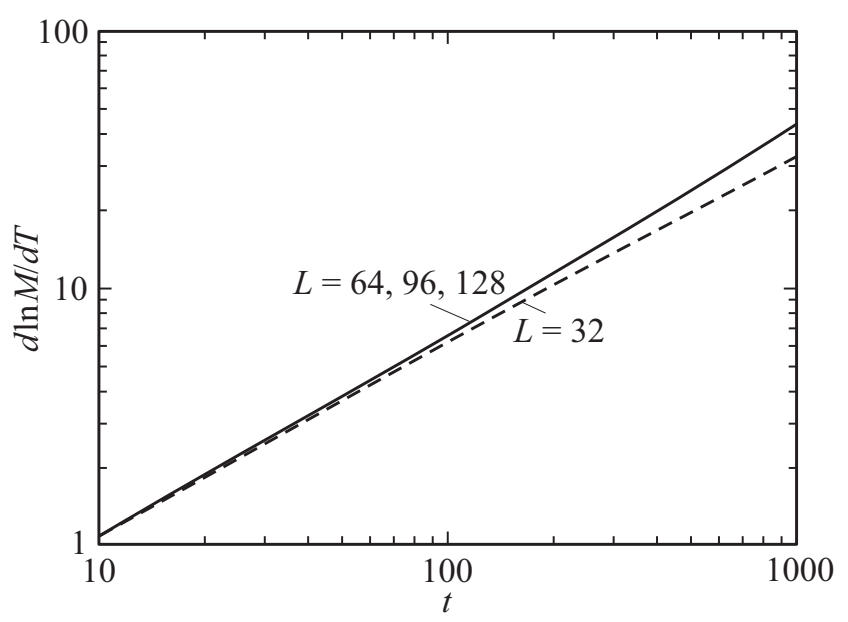

Рис. 4. Зависимость производной логарифма намагниченности от времени в точке фазового перехода для всех линейных размеров $L$. 


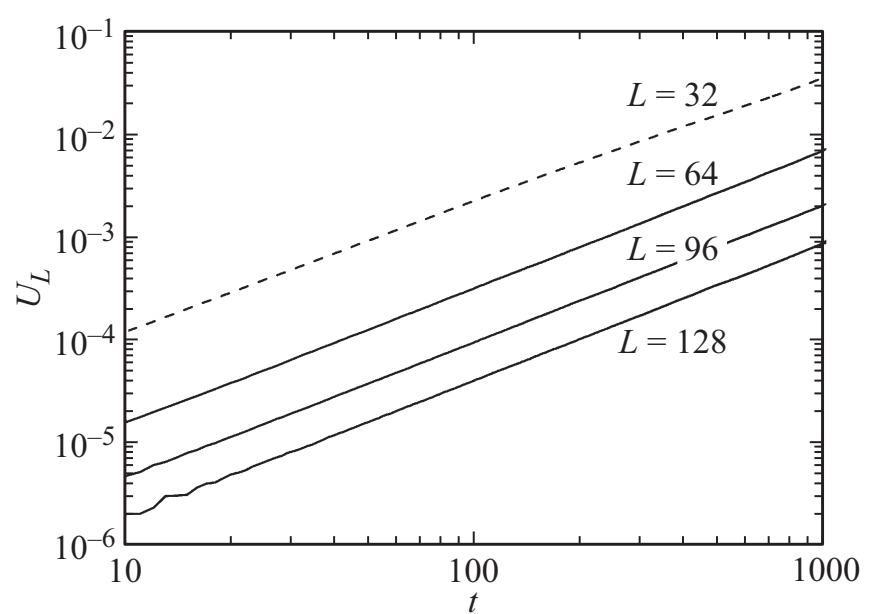

Рис. 5. Зависимость куммулянта Биндера от времени в точке фазового перехода для всех линейных размеров $L$.

зависимости для $L=32$ значительно от них отличаются, и в характере их поведения наблюдается значительное отклонение от скейлингового поведения для значений времени $t \ll 1000$. В результате графики для $L=32$ не представляют собой прямую линию в двойном логарифмическом масштабе даже в точке фазового перехода.

Несколько иная ситуация с графиком зависимости куммулянта Биндера от времени. Поскольку величина самого куммулянта невелика, различие в зависимостях для разных линейных размеров проявляется достаточно хорошо. Построенные по формуле (4) линии аппроксимации для значений $L=64,96$ и 128 не совпадают между собой, но имеют практически одинаковый угол наклона. Для $L=32$, как и в случае с рис. 3 и 4, не реализуется скейлинговое поведение и его график не является прямой линией в двойном логарифмическом масштабе.

Анализ графиков показал, что степенное скейлинговое поведение исследованной системы для линейных ращзмеров $L=64,96$ и 128 реализуется с момента времени порядка $t=150$. Поэтому аппроксимации всех кривых проводились в интервале времени $t=[200 ; 1000]$. Логарифмическая производная в точке фазового перехода вычислялась аппроксимацией методом наименьших квадратов по пяти зависимостям намагниченности от времени, построенным при указанных в табл. 1 температурах. Отметим, что в работе [8] для вычисления логарифмической производной нами использовались три зависимости намагниченности от времени.

Таблица 3. Литературные данные

\begin{tabular}{c|c|c|c}
\hline Параметр & {$[11]$} & {$[12]$} & {$[13]$} \\
\hline$T_{c}$ & $1.344(2)$ & $1.355(2)$ & $1.347(1)$ \\
$\beta$ & $0.21(2)$ & - & $0.25(2)$ \\
$v$ & $0.55(2)$ & $0.55(2)$ & $0.56(2)$
\end{tabular}

В результате аппроксимации полученных данных методом наименьших квадратов в интервале времени $t=[200 ; 1000]$ по формулам (2)-(4) были рассчитаны значения индексов $c_{1}, c_{l 1}$ и $c_{U}$. Их значения позволили рассчитать величины статического критического индекса $\beta$, статического критического индекса радиуса корреляции $v$ и динамического критического индекса $z$. Все полученные нами результаты приведены в табл. 2.

Как видно из таблицы, значения критических индексов и критических температур для линейных размеров $L=64,96$ и 128 в пределах погрешности совпадают между собой, а значения для $L=32$ сильно от них отличаются. Строго говоря, не совсем корректно говорить о критических индексах для $L=32$, так как для этого размера скейлинговое поведение в полной мере не реализуется.

Из-за сильного влияния размерных эффектов для $L=32$ невозможно точно определить значения критической температуры и критических индексов, соответственно результаты для этой системы необходимо исключить из рассмотрения. При этом для систем с линейными размерами $L=64,96$ и 128 не наблюдается заметного влияния размерных эффектов на получаемый результат.

В табл. 3 приведены литературные данные результатов исследований статических критических свойств обсуждаемой модели [11-13]. Сравнение табл. 2 и 3 показывает, что полученные нами результаты для линейных размеров $L=64,96$ и 128 хорошо согласуются с результатами этих работ. Значение динамического критического индекса для полностью фрустрированной модели Изинга получено нами впервые и близко к теоретически предсказанному для анизотропных магнетиков $(z=2$, модель $A,[6])$. Отличие от значения $z=2$ может быть объяснено влиянием фрустраций, но для окончательного ответа на этот вопрос необходимы дальнейшие исследования критической динамики фрустрированных систем.

\section{5. Заключение}

Результаты работы демонстрируют эффективность применения метода коротковременной динамики к изучению критических свойств трехмерных моделей с фрустрацией. Достоинством данного метода является то, что он позволяет в рамках одного численного эксперимента получить не только значение динамического критического индекса, но и значения статических критических индексов и критической температуры. Кроме того, при таком подходе не проявляется критическое замедление, поскольку пространственный радиус корреляции остается небольшим в коротковременном отрезке даже вблизи критической точки [7]. Показано, что для полностью фрустрированной модели Изинга с линейным размером от $L=64$ и более отсутствует влияние конечных размеров на получаемый результат. 


\section{Список литературы}

[1] A. Jaster, J. Mainville, L. Schulke, B. Zheng, E. Print. E-Print arXiv: cond-matt/9808131 v1 (1998).

[2] B. Zheng. Physica A 283, 80 (2000).

[3] V.V. Prudnikov, P.V. Prudnikov, B. Zheng, S.V. Dorofeev, V.Yu. Kolesnikov. Prog. Theor. Phys. 117, 973 (2007).

[4] E.V. Albano, M.A. Bab, G. Baglietto, R.A. Borzi, T.S. Grigera, E.S. Losear, D.E. Rodriguez, M.L. Rubic Puzzo, G.P. Saracco. Rep. Prog. Phys. 74, 026501 (2011).

[5] А.К. Муртазаев, В.А. Мутайламов. ЖЭТФ 143, 695 (2013).

[6] P.C. Hohenberg, B.I. Halperin. Rev. Mod. Phys. 49, 435 (1977).

[7] H.K. Janssen, B. Schaub, B. Schmittmanm. Z. Phys. B 73, 539 (1989).

[8] В.А. Мутайламов, А.К. Муртазаев. Письма в ЖЭТФ 102, 56 (2015)

[9] J. Villain. J. Phys. C 10, 1717 (1977).

[10] D. Blankschtein, M. Ma, A. Nihat Berker. Phys. Rev. B 30, 1362 (1984).

[11] А.К. Муртазаев, И.К. Камилов, М.К. Рамазанов. ФТТ 47, 1125 (2005).

[12] H.T. Diep, P. Lallemand, O. Nagai. J. Phys. C 18, 1067 (1985).

[13] L.W. Bernardi, K. Hukushima, H. Takayama. J. Phys. A 32, 1787 (1999). 